\title{
Thrombolytiques : la génération des chimères
}

Des premiers essais thérapeutiques de la streptokinase dans les thromboses artérielles à la conception actuelle de thrombolytiques chimères produits par génie génétique, cet article illustre de manière probante le chemin parcouru.

\section{Frédéric Caillaud Cadre de recherche.}

\section{RÉFÉRENCES \\ 1. Sainsous J, Richard JL, Lecomte T, Tanie- lan P, Serradimigni A. Combien de malades arrivent à temps ? Résultats d'une enquête nationale ENIM84. Colloque international sur la thrombolyse. Paris, 1985.}

2. Collen D. Tissue type plasminogen activator. Drugs $1986 ; 31: 1-5$.

3. Dano K, Andreasen A, Grondahl-Hansen J, Kristensen P, Nielsen LS, Skriver L. Plasminogen activators, tissue degradation and cancer. Adv Cancer Res 1985 ; 44 : 139-266.

4. Lijnen HR, Zamarron C, Blaber M, Winkler ME, Collen D. Activation of plasminogen by pro-urokinase. J Biol Chem 1986 ; 261 : 1253-8.

5. Verstraete $\mathbf{M}$, Bory $\mathbf{M}$, Collen $\mathrm{D}$, et al. Randomised trial of intravenous recombinant tissue-type plasminogen activator versus intravenous streptokinase in acute myocardial infarction. Lancet 1985 ; i : 842-7.

6. Beecham. European patent 8232228 .

7. Beecham Australian patent 8334498 .

8. Beecham Australian patent 8334499 .

9. Beecham European patent 8230801 .

10. Beecham European patent 8210262 .

NDLR. Les références 6 à 10 correspondent à des brevets déposés.

\section{ADRESSE} 568
F. Caillaud : Genetica, 160, quai de Polangis, gène circulant et certains activateurs du plasminogène s'adsorbent à sa surface, formant un complexe ternaire cyclique avec la fibrine (un des composants majeurs du thrombus) [2]. Le plasminogène est alors efficacement activé en une enzyme protéolytique, la plasmine, qui va dégrader la fibrine et provoquer la lyse du thrombus (figure 1). Les thrombolytiques, ou fibrinolytiques, sont des sérinesprotéases capables d'activer, avant tout, le plasminogène en plasmine. Ces produits sont classés en trois "générations", selon leur date d'apparition sur le marché. Les activateurs du plasminogène de première génération (streptokinase ou urokinase) sont des produits naturels qui, activant le plasminogène circulant et celui adsorbé sur la fibrine, ont pour défaut principal l'induction fréquente de syndromes hémorragiques. Ceux de deuxième génération (activateur tissulaire du plasminogène : tPA et pro-urokinase) sont des produits naturels dont l'affinité pour le plasminogène fixé sur la fibrine est plus grande. Quant aux activateurs du plasminogène de troisième génération, ce sont des chimères issues de modifications chimiques ou génétiques d'activateurs de première ou de deuxième génération.

\section{Première génération}

Il s'agit avant tout de deux produits : la streptokinase et l'urokinase [3]. La streptokinase, d'origine bactérienne, expose, en cas de traitements répétés, à des accidents allergiques en raison de son 
immunogénicité. Son mécanisme d'action est complètement différent de celui des activateurs du plasminogène des eucaryotes. En effet, n'ayant pas d'activité en elle-même, cette molécule doit réaliser un complexe avec du plasminogène, générant de la plasmine, après une série de coupures protéolytiques. L'urokinase (PM: 54000 ; demi-vie : 6 à 8 minutes) dont le mécanisme d'activation du plasminogène est classique (figure 1), est purifiée traditionnellement à partir d'urine humaine (60-100 ng/ml). Compte tenu de cette source, un traitement par l'urokinase (environ 7000 francs) revient trois fois plus cher que celui à base de streptokinase. Jusqu'à présent, le traitement médical d'urgence de l'infarctus du myocarde a reposé sur ces deux médicaments. Or, ils ont en commun un inconvénient majeur, celui d'activer le plasminogène libre. Ceci a pour effet d'augmenter l'activité protéolytique circulante par l'intermédiaire de la plasmine et d'entraîner, après saturation des inhibiteurs spécifiques, une dégradation très importante du fibrinogène, des facteurs $V$ et VIII et d'induire un risque hémorragique majeur. En raison des effets secondaires, le traitement ne peut être effectué qu'en milieu hospitalier spécialisé.

\section{Deuxième génération}

Là encore, ce sont deux sérinesprotéases : la pro-urokinase et le tPA [3]. Si les premiers travaux sur l'urokinase datent de 1955, ceux sur la pro-urokinase ont débuté dans les années 1970. La pro-urokinase (PM: 54000; demivie : 3 à 5 minutes) est une protéine monochaîne, précurseur de l'urokinase (figure 1). Son pouvoir intrinsèque d'activation du plasminogène est égal à celui de l'urokinase, mais il est physiologiquement inhibé dans la circulation sanguine [4]. Cette inhibition pourrait être levée par la fibrine du thrombus (bien que, paradoxalement, la pro-urokinase n'ait pas d'affinité particulière pour la fibrine) ce qui limiterait fortement $\mathrm{m} / \mathrm{s} n^{\circ} 10$ vol. 2, décenbre 86 l'effet systémique désastreux des activateurs du plasminogène de première génération. Cette molécule très prometteuse est en cours d'expérimentation clinique.

Décrit pour la première fois en 1964, le tPA ne représente qu'une infime partie des protéines tissulaires (1 à $2 \mathrm{mg}$ pour $8 \mathrm{~kg}$ d'utérus humain) ou circulantes (concentrations nanomolaires) et il a fallu attendre 1979 pour obtenir du tPA réellement purifié. Immunologiquement différent de l'urokinase, il est sécrété, entre autre, par les cellules de l'endothélium vasculaire sous la forme d'un précurseur monochaîne (PM: 70000) inactif dont la maturation est représentée en figure 1. La chaîne A porte les structures responsables de son affinité pour la fibrine, et la chaîne $B$ le site catalytique habituellement retrouvé dans les sérines-protéases. C'est à un chercheur belge de l'université de Louvain (D. Collen) que l'on doit une grande partie des travaux ayant abouti à la définition des propriétés du tPA, à savoir : une forte affinité pour la fibrine, la nécessité d'un cofacteur tel que la fibrine pour activer le plasminogène, une demi-vie systémique très courte ( 3 à 5 minutes) en raison de la neutralisation par les inhibiteurs spécifiques et de la captation hépatique et l'absence

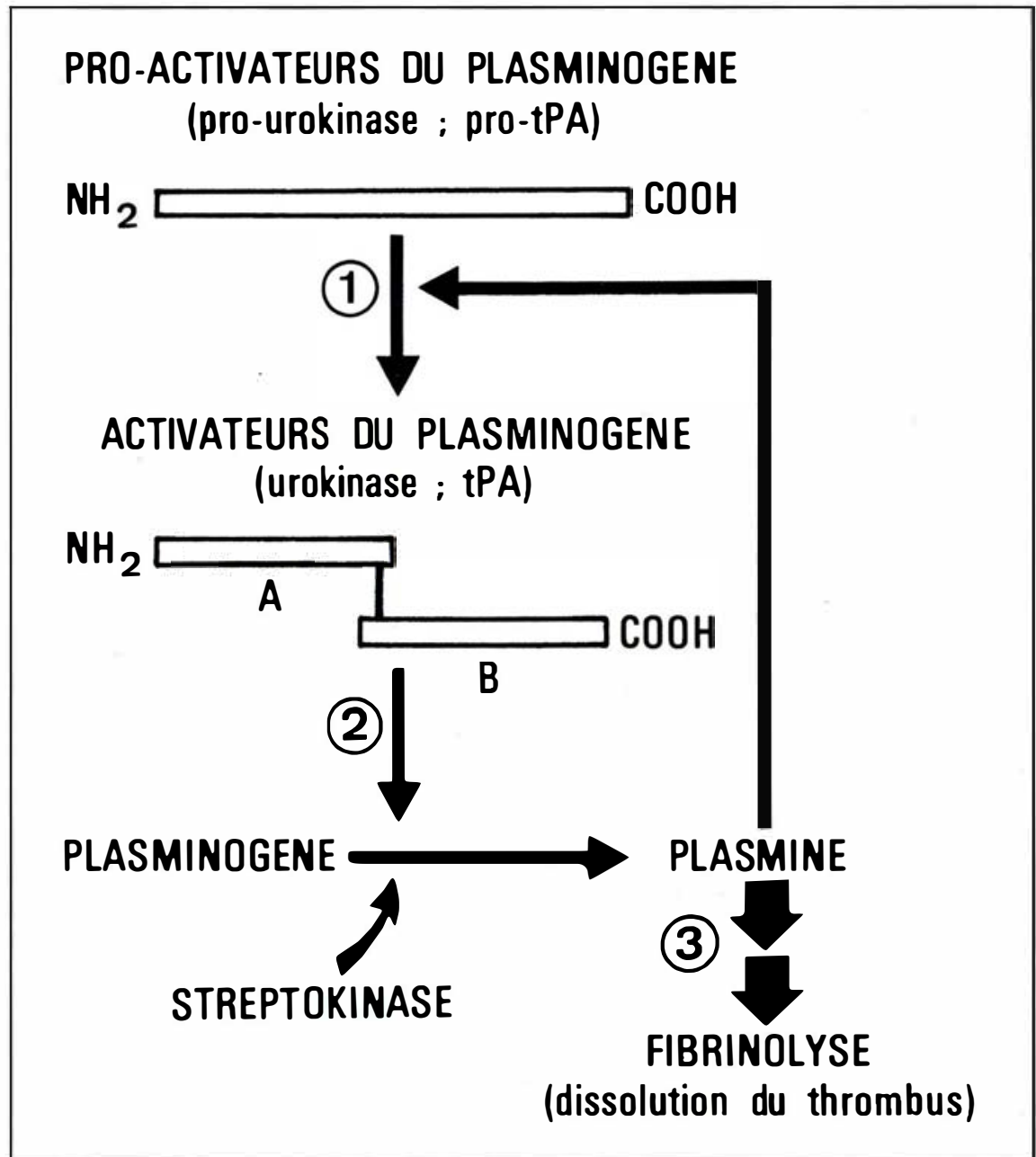

Figure 1. Maturation et mode d'action des activateurs du plasminogène. Des traces de plasmine clivent l'unique chaîne polypeptidique ( $\square$ ) du proactivateur du plasminogène, générant un produit actif constitué de deux chaînes polypeptidiques reliées par un pont disulfure. Le site actif est situé dans la chaîne B. Celui-ci va activer le plasminogène inactif en plasmine active qui pourra analyser la fibrine du thrombus et entretenir la réaction numéro 1. 


\section{RÉFÉRENCES}

11. Sevilla CL, Mahle NH, Boylan CM, Callewaert DM. Plasminogen activator-anti-human fibrinogen conjugate. Biochem Biophys Res Commun 1985 ; 130 : 91-6.

12. Bode C, Matoneda GR, Hui KY, Haber E. Antibody-directed urokinase : a specific fibrinolytic agent. Science $1985 ; 229$ : 765-7.

13. Reboud-Ravaux M. Les activateurs du plasminogène : aspects généraux et développements récents. Biochimie 1985 ; 67 : 1197-216.

14. Van Zonneveld AJ, Veerman H, Pannekoek $\mathrm{H}$. Autonomous functions of structura domains on human tissue-type plasminogen activator. Proc Natl Acad Sci USA 1986 ; 83 : 4670-4.

15. Klausner A. Researchers probe second generation tPA. Biotechnology $1986 ; 4$ : 706-11.

16. Robbins KC, Tanaka Y. Covalent molecular weight 92000 hybrid plasminogen activator derived from human plasmin aminoterminal and urokinase carboxyl-termina domains. Biochemistry 1986 ; 25 : 3603-11.

17. Sobel BE, Fields LE, Robison AK, Keith A, Fox A, Sarnoff S. Coronary thrombolysis with facilitated absorption of intramusculary injected tissue-type plasminogen activator. Proc Natl Acad Sci USA 1985; 82 : 4258-62.

18. Keith A, Fox A, Robison A, Knabb R, et al. Prevention of coronary thrombosis with sub thrombolytic doses of tissue-type plasminogen activator. Circulation $1985 ; 72$ : 1346-54. d'antigénicité. Ces caractères devaient en faire une molécule très spécifique n'entraînant pas d'effet systémique. Malheureusement, en traitement curatif (injection intra-veineuse de 60 à $100 \mathrm{mg}$ de tPA), il y a une certaine fibrinogénolyse, toutefois très inférieure à celle obtenue avec la streptokinase [5]. Le tPA reperméabilise les coronaires dans 60 à $70 \%$ des cas, alors que la streptokinase, dans les mêmes conditions, n'y parvient que chez $45 \%$ des malades. $\mathrm{Si}$ certains ont affirmé que lors de ces essais comparatifs, la streptokinase n'était pas utilisée dans les conditions optimales, il semble que le tPA soit le produit se rapprochant le plus de la définition de l'activateur du plasminogène idéal. Le tPA nécessaire à la réalisation des essais cliniques a été produit par la société américaine Genentech en utilisant les méthodes du génie génétique*. Il devrait être commercialisé (8 à 10000 francs la dose), d'ici un à deux ans.

Dès que les propriétés des activateurs du plasminogène de deuxième génération ont commencé à être connues, de nombreux laboratoires pharmaceutiques et universitaires se sont intéressés à ces produits. Très rapidement, on s'est aperçu que, bien qu'ils soient supérieurs à ceux de première génération, ces fibrinolytiques n'étaient pas parfaits (absence d'affinité vraie pour la fibrine de la pro-urokinase ; effet systémique modéré du tPA). La quête du fibrinolytique idéal a donc repris et c'est ainsi que sont nés les activateurs du plasminogène de troisième génération. A ce stade, il est intéressant de noter l'accélération considérable de cette recherche dans la mesure où aucun des activateurs de deuxième génération n'étant encore sur le marché, on teste déjà un certain nombre de molécules de troisième génération.

\section{La génération des chimères}

Les travaux se sont concentrés sur quatre concepts :

(a) Trouver un nouvel activateur naturel plus performant que ceux déjà étudiés. Si l'on s'en tient aux molécules sérieusement identifiées et caractérisées, capables d'activer le plasminogène, il semble que ce produit n'ait pas encore été découvert.

(b) Augmenter la demi-vie des activateurs du plasminogène, afin de diminuer la dose thérapeutique et donc le coût du traitement. Le laboratoire Beecham, dès 1980, a abordé cette voie et c'est le groupe le plus avancé dans ce domaine. Plusieurs idées directrices ont prévalu. La première était de bloquer réversiblement le site actif d'un activateur du plasminogène parr un groupement capable d'être progressivement hydrolysé, tout en conservant au maximum les propriétés originales, comme l'affinité pour la fibrine (figure $2 A)[6]$. Le blocage par acylation, simple à réaliser, est intéressant biologiquement car la demi-vie des activateurs peut être multipliée par 50 à 100, notamment par élimination des interactions avec les inhibiteurs spécifiques. Cependant, pour obtenir une déacylation totale et une action rapide,
B) tPA (B)-plasmine (A) tPA (A)-uPA (B) tPA (A)-plasmine (B) tPA (B)-acyl-plasmine (A) uPA (B)-plasmine (A) uPA-albumine uPA-plasmine acyl-uPA-plasmine

Figure 2. Exemples de couplages réalisés par le laboratoire Beocham, afin d'augmenter la demi-vie des activateurs du plasminogène. La lettre entre parenthèses indique la chaîne polypeptidique utilisée lors du couplage. TPA : activa-

- Voir le lexique dans ce numéro, p. 579.

* Voir $\mathrm{m} / \mathrm{s} n^{\circ} 1$, vol. 2, p. 51. teur tissulaire du plasminogène; uPA : urokinase; SK : streptokinase. 
il semble qu'il faille injecter plus de produit. Jusqu'à présent, seul le complexe plasminogènestreptokinase-acylée [9] a été réellement étudié puisque les essais cliniques de phase III sont en cours. Il semble que ce produit soit responsable d'effets systémiques et soit hautement immunogène. La deuxième idée était de créer par couplage chimique, une protéase fibrinolytique hybride dont l'une des deux chaînes peptidiques serait liée de façon réversible à une protéine ayant une longue demi-vie et si possible un rôle dans la fibrinolyse (figure 2B) [7, 8]. D'autres laboratoires ont tenté des couplages par des procédés différents avec un succès variable. La troisième idée était de modifier la glycosylation de la molécule d'activateur (principalement le tPA). Si l'élimination de toute glycosylation n'entraîne pas d'effet biologique majeur, sa dégradation partielle semble augmenter la demi-vie et diminuer l'activité [10]. Une autre façon de modifier cette glycosylation consiste à faire exprimer du tPA par recombinaison génétique dans divers types de cellules animales. Quelle que soit la méthode employée, l'augmentation de la demi-vie est très souvent liée à une diminution non négligeable de l'activité fibrinolytique et à un allongement parallèle de la durée des effets secondaires. On s'attendait à une réduction de la dose injectée et du coût du traitement... il semble que ce ne soit pas le cas. Seules des études comparatives sérieuses in vivo permettront de conclure.

(c) Augmenter par modifications chimiques la spécificité de l'activateur (pharmacoguidage) afin d'éviter les effets systémiques. La plupart des travaux ont porté sur l'urokinase. Il s'est agi de préparer des anticorps polyclonaux antifibrogène [11] ou des anticorps monoclonaux dirigés contre les sept acides aminés terminaux de la chaîne $\beta$ de la fibrine humaine [12] et de les coupler de façon covalente à l'urokinase. Ces conjugués sont plus stables et, dans le deuxième cas, il y aurait $m / s n^{\circ} 10$ vol. 2, dicembre 86 potentialisation par un facteur 100 de l'activité fibrinolytique in vitro. Il reste à confirmer ces propriétés, in vivo, et à résoudre le problème du prix de traitement en raison de la nécessité d'une grande quantité d'anticorps qui, de plus, seront certainement immunogènes.

(d) Créer par génie génétique une protéine chimérique au profil idéal. C'est actuellement la voie la plus explorée. Nous relaterons surtout les travaux réalisés sur le tPA, car c'est l'activateur le plus étudié. La chaîne A du tPA (figure 3) présente deux structures caractéristiques de 80 acides aminés : les "kringles". En amont, se trouve une structure tertiaire typique des facteurs de croissance comme l'EGF (facteur de croissance épidermique) et une autre région, en forme de doigt. Ces différentes structures étant codées par des exons différents**, la première étape a été d'essayer d'associer une fonction autonome à chacune d'entre elles [14, 15]. Ainsi, la structure en doigt, non présente dans l'urokinase, semble claire- ment jouer un rôle dans l'affinité du tPA pour la fibrine. Mais elle n'est pas la seule. Il semble établi que le kringle 2, également absent dans l'urokinase, influence la fixation sur la fibrine ainsi que la stimulation de l'activité du tPA par la fibrine. Les deux kringles du tPA étant très homologues, ces propriétés ne peuvent résulter que de subtiles différences. De plus, l'élimination de ces kringles dans diverses sérines-protéases, entraîne, in vitro, une importante diminution de reconnaissance par leurs inhibiteurs spécifiques. Le rôle de la structure de type EGF n'est pas encore élucidé. Les fonctions associées à certaines structures (kringle, doigt) étant identifiées et reliées à des domaines indépendants de la molécule, il devenait envisageable de les prélever par génie génétique pour les associer à des molécules démunies de ces fonctions. Pour cela, il suffit théoriquement de relier, par exemple, le gène de l'urokinase et le fragment d'ADN codant littéralement pour le doigt du tPA. En fait, la structure des protéines étant due

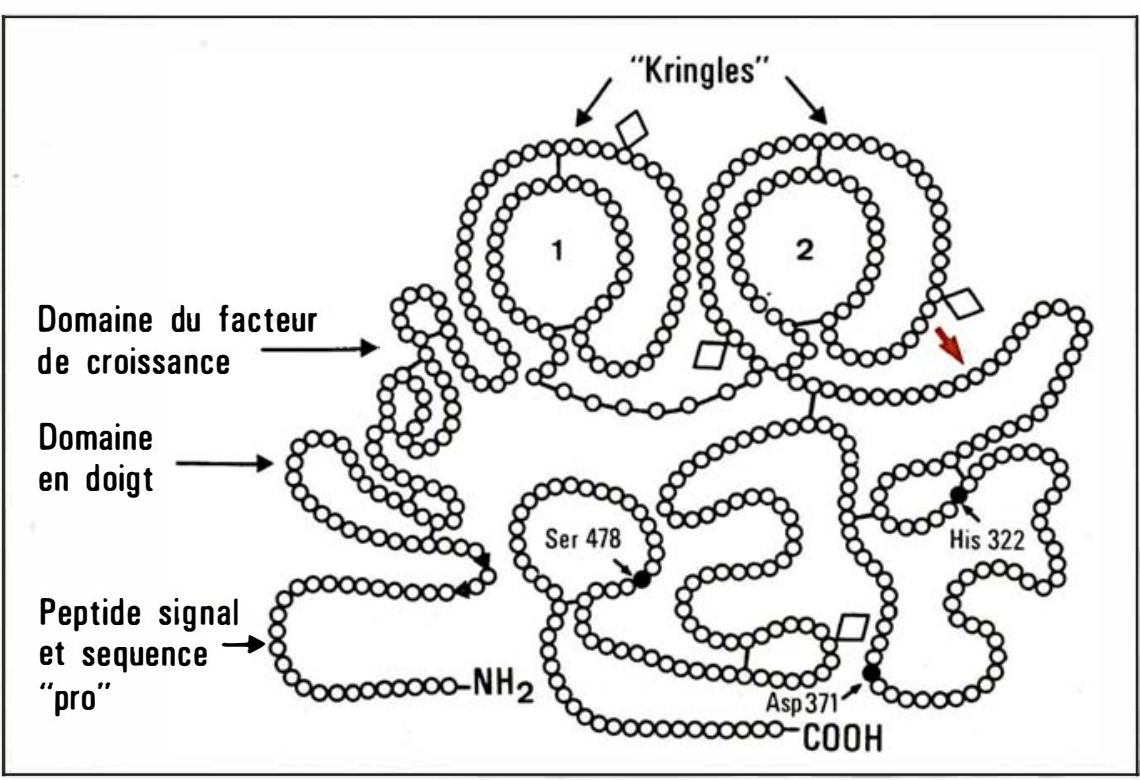

Figure 3. Représentation schématique du précurseur du tPA humain [13]. Les acides aminés sont représentés par des cercles, les ponts disulfures par des barres, les sites potentiels de glycosylation par des losanges, les trois acides aminés importants du site catalytique par leurs noms et leurs positions. La flèche rouge indique le site de coupure conduisant à la formation des deux chaînes du tPA. La compréhension des fonctions de chaque domaine du tPA devrait permettre de développer une troisième génération d'activateurs du plasminogène, plus performante. 
à la convergence d'un certain nombre de facteurs (environnement, séquence...) mals connus actuellement, il est peu probable que soit obtenue dans l'exemple cité une augmentation d'affinité pour la fibrine. Par contre, il y a de fortes chances pour que cette addition entraîne une variation de conformation, d'activité et d'immunogénicité de la molécule d'urokinase, par modification de la structure tertiaire. Un certain nombre de laboratoires sont en train de réaliser ce genre de construction, tout en ayant conscience du caractère aléatoire de leur démarche. Récemment, Robbins $e t$ coll. [16] ont associé équimolairement, par oxydation des ponts disulfures, la chaîne A du plasminogène ( 5 kringles) et la chaîne $B$ de l'urokinase contenant le site actif. Il semble que la stimulation par la fibrine et l'affinité pour cette dernière aient augmenté significativement. La voie est donc ouverte. Un intéressant développement consisterait donc à fusionner le gène d'un anticorps anti-fibrine et celui de l'urokinase, de façon à les exprimer ensemble, augmentant ainsi l'efficacité du complexe. On peut également concevoir de créer un anticorps hybride, ayant une double spécificité pour le tPA et la fibrine, permettant une localisation élective du tPA sur le thrombus. Concrètement, pour terminer, un laboratoire japonais a modifié par mutagenèse dirigée un acide aminé du site actif de la pro-urokinase, la rendant aussi active que le tPA. Le coût de production de la pro-urokinase chez Escherichia coli étant beaucoup moins élevé que celui du tPA dans les cellules animales*, on devine l'intérêt industriel de ce développement.

S'il est envisageable de diminuer artificiellement le niveau des inhibiteurs endogènes des différents fibrinolytiques ou de stimuler la production et la sécrétion endothéliale du tPA in vivo, il est également possible, à partir des fibrinolytiques existants, de définir une nouvelle formulation, afin de créer une amélioration thérapeutique.
Genentech, la société dont les travaux sont très avancés sur le tPA, propose d'injecter du pro-tPA, car celui-ci n'étant activé que sur le thrombus ne devrait pas induire d'effet systémique. Collen pense mimer le processus de la thrombolyse in vivo en associant $3 \mathrm{mg}$ de pro-urokinase et $10 \mathrm{mg}$ de tPA (au lieu de 60 à $100 \mathrm{mg}$ en monothérapie). In vivo, il obtient un effet synergique. Les Soviétiques ont couplé du nitroprussiate de sodium à de l'urokinase : la demivie est augmentée et le complexe, encore actif à $85 \%$, est en plus vasodilatateur. Enfin, une approche très prometteuse consiste en l'injection intra-musculaire précoce de tPA par le malade ou un de ses proches. L'adjuvant mis au point à cette intention cumulerait plusieurs avantages : augmentation de la vitesse d'adsorption du tPA sans induction d'hématome local ; dissociation du tPA de ses inhibiteurs naturels ; inhibition de l'agrégation plaquettaire ; relâchement des muscles lisses. Par sa précocité, cette solution pourrait limiter, sinon éviter, les complications dues à la constitution d'une thrombose coronaire [17].

En conclusion, il faut savoir que les fibrinolytiques sont ou seront, dans un avenir très proche, tous produits par génie génétique. S'il est difficile de prévoir quelle sera la meilleure approche parmi les voies exposées dans cet article, il est possible sans trop de risques d'affirmer que le thrombolytique idéal est à notre portée. Cela est d'autant plus important que ses indications ne se limiteront pas aux infarctus du myocarde, mais s'étendront évidemment aux embolies pulmonaires, aux thromboses veineuses et peut-être, quoique plus difficilement, aux accidents vasculaires cérébraux. On peut envisager aussi une indication préventive [18]. Il est probable que les études réalisées à propos des fibrinolytiques tels que le tPA ou l'urokinase auront également des retombées très larges, dans la mesure où ces molécules jouent un rôle dans l'invasion par des cellules cancéreuses et le remodelage tissulaire

\section{Summary}

Considerable interest in plasminogen activators as human thrombolytic drugs has stimulated rapid biotechnology progresses. These enzymes have been classified in three distinct groups : 1) urokinase and streptokinase which do not interact with fibrin, 2) prourokinase and tissueplasminogen activator which interact with fibrin, 3) chemically or genetically modified plasminogen activators which derive from the above two groups. This review presents the molecular and structural properties of plasminogen activators and how adjuncts could increase plasminogen activation efficiency. Related physiological, pathological and therapeutic aspects are discussed.

\footnotetext{
- Voir le lexique dans ce numéro p. 579.
}

\section{TIRÉS A PART}

F. Caillaud : Genetica, 160, quai de Polangis, 94340 Joinville-le-Pont. 